\title{
Selected vitamins and quality of life in menopausal women
}

\author{
Paweł Milart, Ewa Woźniakowska, Wojciech Wrona \\ $3^{\text {rd }}$ Chair and Department of Gynaecology, Medical University of Lublin, Lublin, Poland
}

\begin{abstract}
The article presents the results of the latest studies on the correlation between the levels of selected vitamins, their bio-availability and their influence on bodily tissues and the quality of life in menopausal women. It also discusses the correlations between vitamin concentrations and the incidence of diseases characteristic of the transitional period, which affects the quality of life.
\end{abstract}

Key words: menopause, vitamins, quality of life.

\section{Vitamin B group}

The role of compounds from the group of vitamin B cannot be overestimated in the menopause. Folate and vitamins $B_{2}, B_{6}$ and $B_{12}$ in their co-enzymatic forms are all essential in one-carbon metabolism, a network of reactions involving the transfer of one-carbon units. B vitamins in general are cofactors for the enzymes that are involved in the energy-producing metabolic pathways for carbohydrates, fats and proteins. B vitamins play an important role in maintaining the functions of the nervous system $[1,2]$.

Deficiencies in any of these B vitamins can disturb the complex regulatory network maintaining one-carbon metabolism, resulting in a reduced methylation status within the relevant tissue, hyperhomocysteinaemia, and pathological formation of DNA, which, in turn, may contribute to adverse health outcomes in menopause [3, 4].

Although diet fortification programmes have been introduced in many countries, the most common cause of the deficiency of vitamin B compounds in menopause is still an inadequate intake. Among the reasons for insufficient supplementation are increased requirements during the menopause, malabsorption caused by intestinal malfunctions, an increasing number of diseases demanding the use of medicines interacting with vitamin B metabolism and, regarding addictions, alcohol abuse, all significantly affecting the levels of all the substances from the vitamin B group [2].

Cardiovascular diseases and stroke as well as cognitive declines resulting in dementia or Alzheimer's are among the several consequences of the insufficient concentration of vitamins from the B group.
Meta-analyses of earlier observational studies have revealed that lowering homocysteine levels by an acceptable vitamin B intake reduces the risk of stroke by 19-24\% [5].

Numerous studies have proved the correlation between homocysteine and low B-vitamin concentrations as relevant factors in cognitive weakening [6] and dementia [7] in menopause and further in older age. And according to Porter et al. [4], there are clear differences in the results of studies on the relationship between folate as well as B-vitamin levels and the occurrence of cognitive dysfunctions in studied groups of patients depending on the area of study. In the countries where food fortification was not introduced, the evidence of these relationships was much clearer.

Functional dementia consists of many morphological changes in the brain. Reduced brain blood flow, neuronal loss, the presence of Lewy bodies, higher than age-appropriate brain atrophy, thinning of cortical gyri as well as pathophysiological malfunctioning such as the damaged connection between the neurons are some of the changes that might occur.

The results of studies in groups with lower baseline folate status have showed correlations between folate in cognitive dysfunction [8] and cognitive decline [9]; moreover, numerous studies have associated low vitamin $B_{12}$ concentration with cognitive dysfunction [10] and cognitive decline [11] in menopause and in older age.

Similarly, decreased vitamin $\mathrm{B}_{6}$ level was correlated with cognitive dysfunction and cognitive decline [12] with a reduced risk of Alzheimer's disease [13].

The role of vitamin $B_{2}$ seems to be less imperative in cognitive malfunction in menopause and the results of the studies are more controversial. 
Meta-analyses of observational studies have confirmed the associations of elevated homocysteine with bone mineral density (BMD) [14] and fracture risk in menopause $[15,16]$. One meta-analysis even concluded that homocysteine was an independent risk factor related to bone fracture [17]. On the other hand, lower concentrations of folate [14], vitamin $B_{12}$ [18], and vita$\min B_{6}[19,20]$ were associated with low BMD.

Yazdanpanah et al. [21] showed that low vitamin $B_{2}$ intake in menopausal women was related to a 1.8 times increased risk of osteoporotic facture and a 2.6 times increased risk of fragility fractures.

It seems that in the face of the increasing frequency of diseases of the vascular system, disorders of brain functioning and abnormal bone metabolism, the assessment of folate and B vitamins should be included in proper medical care of a woman in menopause.

\section{Vitamin C}

Kim et al. [22] studied the influence of vitamin C intake in 198 menopausal Korean women. In their crosssectional study they showed that vitamin C dietary intake was positively associated with selected features of the bone health status of the participants. BMD was measured at the lumbar spine (L1-4), femoral neck and total hip by means of DEXA (dual energy X-ray absorptiometry). The dietary intake of vitamin $C$ was assessed using a semi-quantitative food-frequency questionnaire. It was developed by the National Health and Nutritional Survey of the Korean population in 1998 to document the previous year's food consumption habits [23]. The $T$-score was derived from a comparison between the measured BMD values and the mean BMD of healthy young females [24]. It was revealed that dietary vitamin C intake was positively associated with the femoral neck and the total hip $T$-score, and the $T$-score represented the risk of osteoporotic fracture during the next 10-year period [25].

Another group from South Korea studied the influence of vitamin C dietary intake on bone health status in 1,196 menopausal women [26]. Differently than in the previous studied population, dietary assessment was evaluated using a 24-h dietary recall and BMD was examined by DEXA. When the studied women were allocated to three subgroups according to their daily vitamin C intake (tertiles), the analysis revealed that their bone mineral density showed a dose effect depending on vitamin $\mathrm{C}$ consumption. The authors attempted to find the association between vitamin $C$ and BMD according to vitamin D status. To assess this relationship the studied women were divided into two groups related to their vitamin D status. In the vitamin D-deficient group, with vitamin D concentration lower than $50 \mathrm{nmol} / \mathrm{l}$, BMD was positively correlated with dietary vitamin C intake. The authors did not observe such an effect in the group with higher than $50 \mathrm{nmol} / \mathrm{l}$ vitamin D concentration.

Vijayakumar et al. [27], in their prospective study, supplemented the diet of 56 menopausal women during a 12-week period with vitamin C (dd $50 \mathrm{mg}$ ) or vitamin $B_{12}$ (dd $0.5 \mathrm{mg}$ ). After the treatment they evaluated cognitive abilities in the studied women by means of the Mini-Mental State Examination (MMSE) [28] and measured serum $\beta$-amyloid 42 , which may be deemed as a biochemical marker of cognitive function. Selective MMSE components such as delayed verbal recall, naming and repetition scores were significantly improved and the $\beta$-amyloid 42 concentration decreased significantly in the group supplemented with vitamin $C$. These alterations were not observed in the vitamin $B_{12}$ supplemented group. The authors concluded that vitamin C supplementation is correlated with a significant improvement in cognitive function among menopausal women when compared with methylcobalamin supplementation.

Menopausal women experience a rapid decline in left ventricular diastolic activity, which may mirror the heart failure occurring in the transitional period more frequently. As elevated markers of reactive oxygen species have been reported in the failing human myocardium [29], Ozemek et al. [30] attempted to determine whether oxidative stress may contribute to the decreased left ventricular diastolic function in oestrogendeficient women compared to premenopausal controls, related in part to reduced NO bioavailability. In their experimental study they compared features of mitral flow and brachial artery flow mediated dilation, a biomarker of NO bioavailability, 20 minutes after the acute infusion of saline in the control group and vitamin $C$ in a concentration of $0.06 \mathrm{~g}$ ascorbic acid/ $\mathrm{kg}$ fat-free mass in the studied group. They concluded that their study provides evidence that oxidative stress contributes to reduced LV diastolic function in menopausal women, possibly by reducing the availability of NO.

\section{Vitamin D}

Very few studies have investigated the association between vitamin D status and quality of life. Polisseni et al. [31] compared the effects of $1 \mathrm{mg}$ oestradiol + $0.5 \mathrm{mg}$ norethindrone acetate, $2.5 \mathrm{mg}$ tibolone and supplemental calcium and vitamin D (control group) on quality of life in symptomatic menopausal women. They found improved overall quality of life in all groups. The use of vitamin D in the control group affected the quality of life of women because vitamin D prevented osteoporosis, cardiovascular diseases, diabetes, cancer, infections, and neurodegenerative diseases, although the dose used in the study was very low (200 IU/day). In another interesting study, Chao et al. [32] confirmed the association between vitamin D status and health 
related-quality of life (HRQOL) among the older population (women and men aged 50 years and over). The authors revealed the importance of vitamin $D$ for the HRQOL. Participants of that study with higher serum vitamin D levels were significantly less likely to report problems with mobility, usual activities, depression and anxiety. The authors suggested that HRQOL scores significantly increased with increasing serum vitamin D levels. They also proved that vitamin D supplementation had a minimal effect on quality of life when used on a short-term basis. Another study suggested similar conclusions to the above-mentioned ones. Lower vitamin D status was related to lower scores of quality of life and self-related health in an older population. It is important to remember that a large part of the association can be statistically explained by physical performance, depressive symptoms or chronic diseases of the participants [33]. Manoy et al. [34] also provided evidence that vitamin D supplementation significantly improves quality of life. They studied 158 menopausal women receiving 40,000 IU vitamin D per week for six months. Quality of life was assessed by means of the Short-Form Health Survey, including the physical health composite score and mental health composite score. The obtained results suggested that 40,000 IU of vitamin D supplemented weekly significantly improved quality of life.

On the other hand, Grimnes et al. [35] concluded that lack of vitamin D did not have an impact on quality of life in menopause: in 297 investigated women treated for 12 months with high-dose (20,000 IU) vitamin D taken twice a week, no effect was found as compared to the standard dose (800 IU). In another study, 218 overweight, menopausal women were randomly assigned to 12 months of weight loss +2000 IU oral vitamin D/ day or weight loss + daily placebo. The results showed that compared to placebo, the women receiving vitamin $D$ did not experience any significant change in depressive symptoms, HRQOL subscales, or overall sleep quality. Surprisingly, women who became vitamin $D$ replete ( $\geq 32 \mathrm{ng} / \mathrm{ml}$ ) showed even deterioration in total sleep quality compared to women who remained $<32 \mathrm{ng} / \mathrm{ml}$ despite supplementation [36].

A few studies have investigated the association between vitamin D supplementation and mortality in general. Eaton et al. [37] revealed a negative association between vitamin $\mathrm{D}$ concentration and mortality in the Women's Health Initiative trial, which was modulated by adiposity and other factors. The authors suggested that especially body fat distribution might play an important role in the impact of low vitamin D concentrations on health. Evidence from observational studies indicated an inverse association of circulating vitamin $\mathrm{D}$ with the risks of death due to cardiovascular disease, cancer, and other causes. Chowdhury et al. [38] indicated that supplementation with vitamin D significantly reduced overall mortality among older adults. Data from 42 randomized clinical trials were meta-analyzed by Zheng et al. [39]. The results of their study suggested that supplementation of vitamin D was effective in preventing overall mortality in a long-term treatment, whereas it was not significantly effective in a treatment with duration shorter than 3 years (compare Chao et al. [32]). Another meta-analysis, done on prospective cohort studies, found a nonlinear decrease in mortality risk as vitamin D increased, with optimal concentrations $75-87.5 \mathrm{nmol} / \mathrm{l}$. The authors suggested, however, that large, prospective, randomized trials were needed to investigate the role of vitamin D supplementation in reducing mortality risk in the general population [40].

\section{Vitamin E}

Yang et al. [41] examined 2130 Scottish menopausal women during the Aberdeen Prospective Osteoporosis Study and found a positive association between serum $\alpha$-tocopherol and BMD at the femoral neck. They did not find, however, any correlation between bone health status and the level of dietary vitamin E supplementation, which was assessed by the accomplished food frequency questionnaires. Thus, they could not prove a biologically meaningful correlation between the dietary intake of tocopherol homologues and bone turnover markers or BMD.

Alpha-tocopherol ( $\alpha$-TP) and gamma-tocopherol $(\gamma$-TP), the two predominant isomers of vitamin $E$, were studied for correlations with bone turnover and artery stiffness markers in 278 menopausal women by Hampson et al. [42]. The authors found a significant negative association between $\alpha$-TP and procollagen type 1 aminoterminal propeptide (P1NP). They, however, did not find any significant association between $\gamma$-TP or $\alpha$-TP $/ \gamma$-TP ratio with $\mathrm{P} 1 \mathrm{NP}$ or C-terminal telopeptide of type 1 collagen. Moreover, the study revealed that P1NP was significantly lower in women with $\alpha$-TP concentrations of more than $30 \mu \mathrm{mol} / \mathrm{l}$. Pulse wave velocity in the studied menopausal women was also significantly associated with $\alpha$-TP $/ \gamma$-TP ratio but not with serum $\alpha$-TP or $\gamma$-TP concentrations. The authors concluded that high serum concentrations of $\alpha$-TP may have a negative effect on bone formation and the balance of $\alpha$-TP and $\gamma$-TP may be an important factor in maintaining arterial compliance in menopause.

Shen et al. [43] did not find any beneficial effects, dependent on vitamin $\mathrm{E}$, on quality of life in menopausal women receiving tocotrienol $300 \mathrm{mg} / \mathrm{d}$ and $600 \mathrm{mg} / \mathrm{d}$, who were compared with the control group. During 12-week supplementation, the women did not profit in physical activity level, nutrient intake or quality of life assessed by the Godin Leisure-Time Exercise Questionnaire, Harvard Willett Food Frequency Questionnaire 
and the Medical Outcomes Study 36-item Short Form Health Survey, respectively.

Environmental factors affecting the quality of life at the time of menopause may adversely influence the diet and, in consequence, vitamin supplementation. Factors that may have a potentially dramatic impact include increased morbidity, problems in family life, changes in appetite and eating habits.

The reported studies support the conclusion that a balanced supply of the selected vitamins might contribute to ameliorating the quality of life in menopausal women.

\section{Disclosure}

The authors report no conflict of interest.

\section{References}

1. Nutrition Working Group, O'Connor DL, Blake J, et al. Canadian consensus on female nutrition: adolescence, reproduction, menopause and beyond. J Obstet Gynaecol Can 2016; 38: 508-554.

2. Porter K, Hoey L, Hughes CF, et al. Causes, consequences and public health implications of low B-vitamin status in ageing. Nutrients 2016; 8: 1-29.

3. Brachet , Chanson A, Demigne $C$, et al. Age-associated B vitamin deficiency as a determinant of chronic diseases. Nutr Res Rev 2004; 17: 55-68.

4. Araujo JR, Martel F, Borges N, et al. Folates and aging: Role in mild cognitive impairment, dementia and depression. Ageing Res Rev 2015; 22: 9-19.

5. Homocysteine Studies Collaboration. Homocysteine and risk of ischemic heart disease and stroke: A meta-analysis. JAMA 2002; 288: 2015 2022.

6. Smith AD. The worldwide challenge of the dementias: A role for B vitamins and homocysteine? Food Nutr Bull 2008; 29: S143-S172.

7. Smith DA, Refsum H. Homocysteine, B vitamins, and cognitive impairment. Annu Rev Nutr 2016; 36: 211-239.

8. Horvat P, Gardiner J, Kubinova R, et al. Serum folate, vitamin B-12 and cognitive function in middle and older age: The HAPIEE study. Exp Gerontol 2016; 76: 33-38.

9. Brown B, Huang MH, Karlamangla A, et al. Do the effects of APOEepsilon4 on cognitive function and decline depend upon vitamin status? MacArthur Studies of Successful Aging. J Nutr Health Aging 2011; 15: 196-201.

10. Moorthy D, Peter I, Scott TM, et al. Status of vitamins B-12 and B- 6 but not of folate, homocysteine, and the methylenetetrahydrofolate reductase C677T polymorphism are associated with impaired cognition and depression in adults. J Nutr 2012; 142: 1554-1560.

11. Morris MS, Selhub J, Jacques PF. Vitamin B-12 and folate status in relation to decline in scores on the mini-mental state examination in the Framingham heart study. J Am Geriatr Soc 2012; 60: 1457-1464.

12. Kado DM, Karlamangla AS, Huang M, et al. Homocysteine versus the vitamins folate, $\mathrm{B} 6$, and $\mathrm{B} 12$ as predictors of cognitive function and de cline in older high-functioning adults: MacArthur Studies of successful aging. Am J Med 2005; 118: 161-167.

13. Corrada MM, Kawas CH, Hallfrisch J, et al. Reduced risk of Alzheimer's disease with high folate intake: The Baltimore Longitudinal Study of Aging. Alzheimer's Dement 2005; 1: 11-18.

14. Gjesdal CG, Vollset SE, Ueland PM, et al. Plasma total homocysteine level and bone mineral density: The hordaland homocysteine study. Arch Intern Med 2006; 166: 88-94.

15. Enneman AW, Swart KM, VanWijngaarden JP, et al. Association between plasma homocysteine and bone density and quality parameters in the elderly. Bone 2012; 50: S134.
16. McLean RR, Jacques PF, Selhub J, et al. Homocysteine as a predictive factor for hip fracture in older persons. N Engl J Med 2004; 350: 2042-2049.

17. Yang J, Hu X, Zhang Q, et al. Homocysteine level and risk of fracture: A meta-analysis and systematic review. Bone 2012; 51: 376-382.

18. Tucker KL, Hannan MT, Qiao N, et al. Low plasma vitamin B12 is associated with lower BMD: The Framingham Osteoporosis Study. J Bone Miner Res 2005; 20: 152-158.

19. Dai Z, Wang R, Ang LW, et al. Dietary B vitamin intake and risk of hip fracture: The Singapore Chinese Health Study. Osteoporos Int 2013; 24 : 2049-2059.

20. McLean RR, Jacques PF, Selhub J, et al. Plasma B vitamins, homocysteine, and their relation with bone loss and hip fracture in elderly men and women. J Clin Endocrinol Metab 2008; 93: 2206-2212.

21. Yazdanpanah N, Zillikens MC, Rivadeneira F, et al. Effect of dietary B vitamins on BMD and risk of fracture in elderly men and women: The Rotterdam Study. Bone 2007; 41: 987-994.

22. Kim DE, Cho SH, Park HM, et al. Relationship between bone mineral density and dietary intake of $\beta$-carotene, vitamin $C$, zinc and vegetables in postmenopausal Korean women: a cross-sectional study. J Int Med Res 2016; 44: 1103-1114.

23. Ahn Y, Lee JE, Paik HY, et al. Development of a semi-quantitative food frequency questionnaire based on dietary data from the Korean National Health and Nutrition Examination Study. Nutr Sci 2003; 6: 173-184.

24. World Health Organization. Assessment of fracture risk and its application to screening for postmenopausal osteoporosis. Report of a WHO Study Group. World Health Organ Tech Rep Ser 1994; 843: 1-129.

25. Badurski JE, Dobreńko A, Nowak NA, et al. Kalkulator RB-10, czyli: Obliczanie indywidualnego, bezwzględnego, 10-letniego ryzyka złamania osteoporotycznego. http://www.pfo.com.pl/KalkulatorPL.html

26. Kim YA, Kim KM, Lim S, et al. Favorable effect of dietary vitamin C on bone mineral density in postmenopausal women (KNHANES IV, 2009): discrepancies regarding skeletal sites, age, and vitamin D status. Osteoporos Int 2015; 26: 2329-2337.

27. Vijayakumar TM, Pavitra K, Muthunarayanan L. Comparative assessment of methylcobalamin and ascorbic acid on cognitive function in post-menopausal women - A randomized, double-blind trial. Contemp Clin Trials Comm 2017; 8: 175-180.

28. Folstein MF, Folstein SE, McHugh PR. "Mini-mental state". A practical method for grading the cognitive state of patients for the clinician. J Psychiatri Res 1975; 12: 189-198.

29. Santos CX, Anilkumar N, Zhang M, et al. Redox signaling in cardiac myocytes. Free Radic Biol Med 2011; 50: 777-793.

30. Ozemek C, Hildreth KL, Groves DW, et al. Acute ascorbic acid infusion increases left ventricular diastolic function in postmenopausal women. Maturitas 2016; 92: 154-161.

31. Polisseni AF, Andrade AT, Ribeiro LC, et al. Effects of a continuouscombined regimen of low-dose hormone therapy (oestradiol and norethindrone acetate) and tibolone on the quality of life in symptomatic postmenopausal women: a double-blind, randomised study. Maturitas 2013; 74: 172-178.

32. Chao YS, Ekwaru JP, Ohinmaa A, et al. Vitamin D and health-related quality of life in a community sample of older Canadians. Qual Life Res 2014; 23: 2569-2575.

33. Rafiq R, Swart KM, van Schoor NM, et al. Associations of serum 25-hydroxyvitamin D concentrations with quality of life and self-rated health in an older population. J Clin Endocrinol Metab 2014; 99: 3136-3143.

34. Manoy P, Yuktanandana P, Tanavalee A, et al. Vitamin D Supplementation Improves Quality of Life and Physical Performance in Osteoarthritis Patients. Nutrients 2017; 9: 799.

35. Grimnes G, Emaus N, Cashman KD, et al. The effect of high-dose vitamin D supplementation on muscular function and quality of life in postmenopausal women - A randomized controlled trial. Clin Endocrinol 2017; 87: 20-28.

36. Mason C, de Dieu Tapsoba J, Duggan C, et al. Repletion of vitamin D associated with deterioration of sleep quality among postmenopausal women. Prev Med 2016; 93: 166-170.

37. Eaton CB, Young A, Allison MA, et al. Prospective association of vitamin D concentrations with mortality in postmenopausal women: results from the Women's Health Initiative (WHI). Am J Clin Nutr 2011; 94: 1471-1478.

38. Chowdhury R, Kunutsor S, Vitezova A, et al. Vitamin D and risk of cause specific death: systematic review and meta-analysis of observational 
cohort and randomised intervention studies. BMJ 2014; 348: g1903. doi: 10.1136/bmj.g1903: 10.1136/bmj.g1903.

39. Zheng Y, Zhu J, Zhou M, et al. Meta-analysis of long-term vitamin D supplementation on overall mortality. PLoS One 2013; 8: e82109. doi: 10.1371/journal.pone.0082109.

40. Zittermann A, Prokop S. The role of vitamin D for cardiovascular disease and overall mortality. Adv Exp Med Biol 2014; 810: 106-119.

41. Yang TC, Duthie GG, Aucott LS, et al. Vitamin E homologues $\alpha$ - and $\gamma$-tocopherol are not associated with bone turnover markers or bone mineral density in peri-menopausal and post-menopausal women. Osteoporos Int 2016; 27: 2281-2290.

42. Hampson G, Edwards S, Sankaralingam A, et al. Circulating concentrations of vitamin $\mathrm{E}$ isomers: Association with bone turnover and arterial stiffness in post-menopausal women. Bone 2015; 81: 407-412.

43. Shen C-L, Wang S, Yang S, et al. A 12-week evaluation of annatto tocotrienol supplementation for postmenopausal women: safety, quality of life, body composition, physical activity, and nutrient intake. BMC Complement Altern Med 2018; 18: 198. 